\title{
Análisis de la importancia de los modelos de gestión como estrategia de mejora continua en los Gobiernos Autónomos Descentralizados Municipales de la Provincia de Cotopaxi
}

\author{
Fecha de recepción: 01-03-2021 • Fecha de aceptación: 10-05-2021 • Fecha de publicación: 10-06-2021
}

Carmen Marlene Choloquinga Escobar Gobierno Autónomo Descentralizado Parroquial Rural de "Chugchilán" carmencholoquingae@gmail.com https://orcid.org/0000-0002-8516-5060

\section{RESUMEN}

Desde el año 2009, con la implementación del Sistema Nacional de Información pública, coordinado por la Secretaría Técnica de Planificación "Planifica Ecuador" que abarca un conjunto organizado en elementos que permiten a la planificación cumplir con las metas en el desarrollo y las finanzas públicas, resulta importante en los Gobiernos Autónomos Descentralizados Municipales que apliquen un modelo de gestión como un instrumento innovador con la visión de reflejar eficiencia y eficacia a la gestión pública por procesos relacionada a resultados; de esta manera, el presente trabajo de tiene como objetivo analizar la importancia de los modelos de gestión como estrategia de mejora continua en los Gobiernos Autónomos Descentralizados Municipales de la Provincia de Cotopaxi.

Se consideraron puntos importantes que afectan al resultado eficiente en la administración pública y la forma que el indicador de las Necesidades Básicas Insatisfechas territoriales tiene un impacto directo con la ejecución de los presupuestos, eje central en atender la demanda de los habitantes. El trabajo se desarrolló mediante la recolección de datos con encuestas aplicadas a los funcionarios responsables del área/unidad de planificación de los gobiernos municipales y documental, el impacto de la aplicación de un modelo de gestión por procesos permite a los municipios enmarquen su acción en estrategias de mejora continua que lleguen a cumplir con el objetivo que adopten una administración publica eficiente con resultados. 
PALABRAS CLAVE: planificación, plan de desarrollo, modelo de gestión, gobiernos autónomos, administración pública, presupuesto.

\section{ABSTRACT}

Since 2009, with the implementation of the National Public Information System, coordinated by the Technical Secretariat of Planning "Planifica Ecuador", which includes a set of organized elements that allow planning to meet the goals in development and public finances, it is important in the Municipal Decentralized Autonomous Governments to apply a management, Thus, the objective of this work is to analyze the importance of management models as a strategy for continuous improvement in the Municipal Decentralized Autonomous Governments of the Province of Cotopaxi.

Important points that affect the efficient result in public administration and the way in which the territorial unsatisfied basic needs indicator has a direct impact on the execution of budgets, the central axis in meeting the demand of the inhabitants, were considered. The work was developed through the collection of data with surveys applied to officials responsible for the planning area/unit of the municipal and documentary governments, the impact of the application of a process management model allows the municipalities to frame their action in continuous improvement strategies that reach the objective of adopting an efficient public administration with results.

KEYWORDS: planning, development plan, management model, autonomous governments, public administration, budget. 


\section{Introducción}

El marco legal del Código Orgánico de Organización Territorial, Autonomía y Descentralización COOTAD (2015) establece que "la descentralización permite transferir de manera obligatoria, progresiva y definitiva competencias con los respectivos recursos financieros, materiales y tecnológicos desde el Gobierno Central hacia los gobiernos provinciales, municipales y parroquiales denominados (GAD's), que actúan con autonomía política, administrativa y financiera".

En el ámbito de procesos, Pepper (2011) considera que la gestión por procesos puede definirse o aplicarse como una forma de enfocar el trabajo, donde se estrecha el mejoramiento continuo de las acciones de una organización mediante la identificación, selección, descripción, documentación y mejora continua en los procesos.

Este modelo es considerado un instrumento estratégico de resultados aplicado en las entidades públicas con una estrategia de eficiencia, en este caso, los municipios en obra pública y prestación de servicios que se canalicen para satisfacer los requerimientos de la población territorial y mejora de la cultura organizacional. En este sentido, el modelo de gestión (Chilán et al., 2016) termina siendo una simple obligación que las instituciones públicas tienen que cumplir; sin embargo, a pesar que los gobiernos municipales deben aplicar el modelo de gestión con indicadores de resultados, evaluación y seguimiento, es una premisa que, de 7 municipios, Sigchos y Saquisilí están rompiendo paradigmas de pasar de un modelo tradicional, a un modelo de herramientas de gestión por resultados.

Para cristalizar la eficiencia de los modelos de gestión por procesos se debe ejecutar los presupuestos de los programas y proyectos planificados que están estrechamente relacionado con el porcentaje de las Necesidades Básicas Insatisfechas (NBI), según la última encuesta de Instituto Nacional de Estadísticas y Censos (2010) la pobreza por NBI en la provincia de Cotopaxi tiene una tasa promedio del $81,73 \%$ correspondiente a los siete cantones, mientras que el 17.93 $\%$ de las personas no son pobres, es decir, cuentan con todos los servicios básicos, sociales y equipamiento correspondiente.

Además, los modelos de gestión (López, 2001) deben manejarse con criterios de competitividad y sustentabilidad, entregar servicios a la ciudadanía a precios razonables y de calidad; la importancia del modelo de gestión se centralizada en las entidades que deben optimizar recursos, procesos enlazados con el recurso económico, tiempo y servicios que se prestan a la ciudadanía y un desempeño de la gestión organizacional (Vidal, 2004).

En ese sentido, el artículo presentado tiene como objetivo analizar la importancia de la aplicación de los modelos de gestión como estrategia de mejora continua en los Gobiernos Autónomos Descentralizados Municipales de la Provincia de Cotopaxi, Ecuador. 


\subsection{Estado del arte}

De acuerdo con la Superintendencia de Ordenamiento Territorial, Uso y Gestión de Suelo (2015), en Ecuador son 221 Gobiernos Municipales y 7 Gobiernos Autónomos Descentralizados Municipales que corresponden a la provincia de Cotopaxi Latacunga, La Maná, Pangua, Pujilí, Salcedo, Saquisilí y Sigchos son entidades públicas como menciona el COOTAD cuentan con independencia de derecho público, autonomía política, administrativa y financiera, se vinculan estrechamente con la normativa de Constitución ecuatoriana y leyes pertinentes que conducen su funcionamiento y administración en todos los ámbitos admisibles en el marco territorial

El 48\% de los municipios no logra consolidar sus instrumentos de planificación, reporte y evaluación que conlleve sus modelos de gestión a ser estratégicos y el $74 \%$ según los datos recopilados los modelos de gestión necesitan ser evaluados y reestructurados. La investigación se orienta a sumar principios ideológicos básicos de la importancia de la definición y aplicación de un modelo de gestión (Fernández, 2001) que se enmarcan al desarrollo territorial público, permitiendo detectar fortalezas y/o debilidades en las entidades y pueda inferir en una evaluación más allá del reporte y publicación de información que refleje el grado de cumplimiento de la planificación estratégica, con los objetivos planteados optimizando su desempaño organizacional.

Cuando hablamos de los Gobiernos Autónomos Descentralizados, de acuerdo con el Código Orgánico Organización Territorial Autonomía Descentralización, en el art. 238 se menciona que estos "constituyen gobiernos autónomos descentralizados las juntas parroquiales rurales, los concejos municipales, concejos metropolitanos, consejos provinciales y consejos regionales, gozarán de autonomía política, administrativa y financiera, y se regirán por los principios de solidaridad, subsidiariedad, equidad interterritorial, integración y participación ciudadana".

En el marco legal que regula el ámbito de acción a los gobiernos municipales, la COOTAD establece en el art. 193 que para la asignación y distribución de recursos a cada gobierno autónomo descentralizado se deberá aplicar un modelo de equidad territorial en la provisión de bienes y servicios públicos que reparte el monto global de las transferencias en dos tramos, de la siguiente manera, tal como lo exponen Chávez \& Otavalo (2018):

a) La distribución de las transferencias a los gobiernos autónomos descentralizados tomará el 2010 como año base y repartirá el monto que por ley les haya correspondido a los gobiernos autónomos en ese año.

b) El monto excedente del total del veintiuno por ciento (21\%) de ingresos permanentes y diez por ciento $(10 \%)$ de ingresos no permanentes restados los valores correspondientes a las transferencias entregadas el año 2010, se distribuirá entre los gobiernos autónomos a través de la aplicación de los criterios constitucionales conforme a la fórmula y la ponderación de cada criterio señalada en este Código.

De conformidad a la normativa el art. 192 del COOTAD y al art. 272 de la Constitución del Ecuador, los criterios de distribución de los recursos a los GAD son los siguientes: 
Considerando el "1. Tamaño de la población 2. Densidad de la población 3. Necesidades básicas insatisfechas jerarquizadas y consideradas en relación con la población residente en el territorio de cada uno de los GAD 4. Logros en el mejoramiento de los niveles de vida 5. Esfuerzo fiscal 6. Esfuerzo administrativo 7. Cumplimiento de metas del plan nacional de desarrollo y del plan de desarrollo del GAD. De cada uno de estos criterios, el COOTAD estipula la ponderación, peso o participación de cada uno de ellos en el monto general a distribuirse, diferenciado por nivel de gobierno".

Los Gobiernos Autónomo Descentralizados, a través de las diferentes entidades de seguimiento y control, trabajan en una administración para reducir indicadores de pobreza de un territorio, los $\mathrm{NBI}$ están estrechamente relacionados con la eficiencia de sus instrumentos de planificación.

Para identificar cómo elaborar un modelo de gestión para un Gobierno Autónomo Descentralizado Municipal nos referimos al art. 128, inciso 3, del COOTAD 2010, que menciona lo siguiente:

"Los diferentes sectores se organizarán, funcionarán y someterán a los principios y normas definidos en el sistema nacional de competencias, sí los modelos de gestión que se desarrollen en los regímenes especiales observarán necesariamente la distribución de competencias y facultades, criterios y normas, contenidas en este Código para los distintos niveles de gobierno".

En este sentido, el modelo de gestión de los Gobiernos Municipales, en concordancia con los planes de desarrollo y ordenamiento territorial (Peñeherrera, 2016), son procedimientos y acciones que encaminan a la implementación de los programas y proyectos que son considerados acorde a las necesidades territoriales para resolver problemas y fortalecer potencialidades a través del espacio de la democracia participativa. Adicional, permite establecer una orientación y un marco de referencia objetiva para actuar ante el diagnóstico situacional de la entidad, encaminado a enlazar esfuerzos estratégicos que identifiquen ejes para proceder y evaluar el alcance de la productividad de la entidad seccional.

De acuerdo a lo anterior, la importancia de los modelos de gestión (Figura 1) tiene que ver con que últimamente varias entidades del sector público han insertado procesos de administración que optimicen recursos con sistemas o modelos de gestión, que reflejen sistemas o flujos de trabajos administrativos que acceden a la variedad de estrategias en el control automático y preciso de los recursos y ocupaciones en una gerencia administrativa de ocupaciones burocráticas (Waissbluth, 2020). 


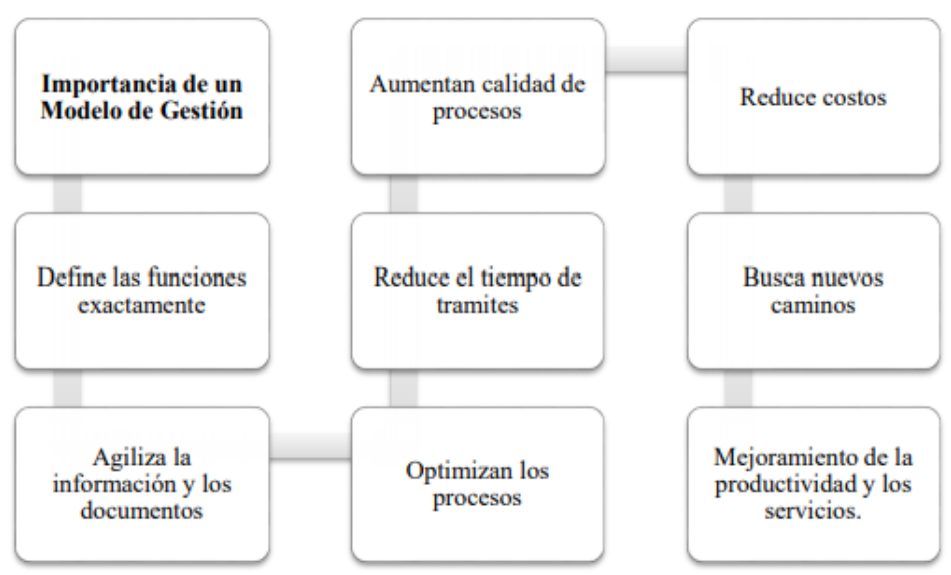

Figura 1. Importancia de un modelo de gestión

Fuente: elaboración propia

El conocimiento de la importancia, aplicado en una entidad, requiere la colaboración de todos los involucrados, ya que se realiza un diagnostico organizacional, se define funciones, agiliza la información y los documentos, optimizan los procesos, reduce el tiempo de trámites, aumentan calidad de procesos, reduce costos, busca nuevos caminos de mejoramiento de la productividad y los servicios con impactos a resultados satisfactorios ante los actores territoriales (Izquierdo, 2015).

Los Gobiernos Autónomos Descentralizados Municipales, al aplicar un modelo de gestión idóneo con enfoque a un modelo por procesos, podrán reflejar estrategias de mejora continua para fortalecer la administración pública (Bañón,1993) acorde a sus competencias, y a su vez permitirá mejorar los servicios a la ciudadanía como a los usuarios de los municipios logrando eficiencia y eficacia en el marco institucional.

\subsection{Estrategias}

Una administración pública eficiente genera mejoras continuas,por lo que una estrategia es una acción que asegura los servicios públicos sean de calidad y se enfoquen en la excelencia de la gestión pública (Peteiro, s.f) de acuerdo con los ejes de acción en los Gobiernos Autónomos Descentralizados. El modelo de excelencia es una adaptación del Modelo CAF (Marco Común de Evaluación o Common Assessment Framework), este permite obtener ventajas estratégicas con valuación cuantitativa y cualitativa, análisis de tendencias, análisis comparativo, intercambio de experiencias, metodologías y forma de aplicación del modelo, reducción de costos e información de apoyo para la toma de decisiones (Flores, 2017).

Son primordiales las directrices y estrategias transversales a cada una de los Gobiernos Autónomos Descentralizados, los que se ven traducidos en normativas, proyectos, metodologías y formación en cada una; y junto con la innovación tienen la posibilidad de conseguir los recursos deseados con la ciudadanía y sociedad. 


\section{Metodología}

El enfoque metodológico aplicado en este estudio fue el mixto, en vista que se utilizó el enfoque cualitativo en base a la revisión documental y cuantitativa (Hernández et al., 2014), aplicando encuestas a los funcionarios del departamento de planificación de los gobiernos municipales.

A través de la utilización de los métodos descriptivo y analítico, se logró profundizar sobre los resultados obtenidos mediante la investigación documental, correspondiente a la información obtenida de la fuente principal que es la Secretaria Técnica Planifica Ecuador, antes SENPLADES, con las estadísticas de la NIB, frente a una administración, Superintendencia de Ordenamiento Territorial, Uso y Gestión del Suelo función de vigilar y controlar los procesos de ordenamiento territorial de todos los niveles de gobierno, y del uso y gestión del suelo, hábitat, asentamientos humanos y desarrollo urbano que realizan los Gobiernos Autónomos Descentralizados municipales y metropolitanos dentro del marco de sus competencias y los Planes de Desarrollo y Ordenamiento Territorial con énfasis al modelo de gestión.

El tipo de muestreo aplicado fue no probabilístico (Scharager, 2001), teniendo como resultado 42 funcionarios encuestados en forma virtual en la plataforma google. De la población en la que se desarrolló el estudio fueron los 7 Gobiernos Municipales de la Provincia de Cotopaxi.

Así también las técnicas utilizadas en el presente trabajo obedecen a la observación del Código Orgánico Organización Territorial Autonomía, Sistematización de Buenas Prácticas Institucionales en el marco del modelo de gestión del Conocimiento (SENPLADES, 2016) y los objetivos de Desarrollo Sostenible (PNUD, S.F) relacionado al Plan Nacional de Desarrollo.

\section{Resultados}

Partiendo de un análisis de los Planes de Desarrollo y Ordenamiento Territorial de los Gobiernos Autónomos Descentralizados Municipales de la Provincia de Cotopaxi acorde al art. 42 del Código Orgánico de Planificación y Finanzas Públicas señala que los contenidos mínimos son: diagnóstico estratégico, propuesta y modelo de gestión y tomando en cuenta lo mencionado se estructuró la siguiente matriz identificando factores internos y externos que se muestran en la Figura 2. 


\begin{tabular}{|c|c|}
\hline $\begin{array}{l}\text { Fortalezas } \\
\text { Los Gobiernos Autónomos Municipales constituyen un } \\
\text { nivel de Gobierno Autónomo Descentralizado. } \\
\text { Disponer de indicadores de gestión }\end{array}$ & $\begin{array}{l}\text { Oportunidades } \\
\text { Articulación con diferentes niveles de Gobierno. } \\
\text { Establecer convenios de gestión interinstitucional o } \\
\text { delegación de competencias. }\end{array}$ \\
\hline $\begin{array}{l}\text { Debilidades } \\
\text { Cultura Organizacional parcial } \\
\text { Desconocimiento de lineamiento de la ejecución de un } \\
\text { modelo de gestión } \\
\text { Débil seguimiento y evaluación } \\
\text { Modelos de gestión por procesos desactualizados }\end{array}$ & $\begin{array}{l}\text { Amenazas } \\
\text { Recortes presupuestarios por parte del Gobierno } \\
\text { Central. } \\
\text { No reducir los indicadores de NBI. }\end{array}$ \\
\hline
\end{tabular}

Figura 2. Matriz de identificación de factores internos y externos

Fuente: elaboración propia

Los modelos de gestión territoriales de los municipios tienen que ser actualizados, reestructurados y proponer nuevas estrategias de articulación vinculadas con los factores acorde a la realidad con el fin de reducir las demandas / necesidades el territorio con y los programas y proyectos ejecutables para beneficio social.

El análisis de la investigación también parte que desde el año 2009, frente a la necesidad de regir, articular e integrar la información de las instituciones productoras de datos, traducida en herramientas que contenga datos que orienten la toma de elecciones, el diseño de políticas públicas y las intervenciones del Estado, está el Proyecto Nacional de Desarrollo y demás aparatos de organización; conforme al Estatuto Orgánico de la Entidad y la normativa que existe. En este entorno, el desarrollo del Sistema de Nacional de Información (2009) implica la responsabilidad de seguir los pasos metodológicos basados en el Modelo de Gestión de la Información del Estado recogido en el documento "Sistema Nacional de Información: Un nuevo modelo de gestión de la información nacional", en el cual la toma de decisiones para la gestión pública se realiza en alguno de los tres niveles organizacionales de gobierno: estratégico, táctico y operativo.

Incluye un modelo de seguimiento (Tabla 1) y evaluación la Secretaria Técnica Planifica Ecuador que permita a los Gobiernos Autónomos Descentralizados el control de la implementación, avance y resultados de su instrumento de planificación, con la finalidad de encontrar las causas y consecuencias del incumplimiento de sus planes, a través de procesos de análisis, indicadores, metas y productos. 
Tabla 1.

Matriz de reporte, evaluación y seguimiento -SOT

\begin{tabular}{|l|l|l|}
\hline Categoría de evaluación & Número & Porcentaje \\
\hline Sí tienen evaluación & 35 & $16 \%$ \\
\hline Actualización y alineación & 42 & $19 \%$ \\
\hline Otras acciones & 38 & $17 \%$ \\
\hline No tienen evaluación & 12 & $5,5 \%$ \\
\hline No reporta & 94 & $42,5 \%$ \\
\hline Total general & 221 & $100 \%$ \\
\hline
\end{tabular}

Fuente: elaboración propia

Como respuesta a un factor adicional a la importancia de la aplicación de un modelo de gestión en los Planes de Desarrollo y Ordenamiento Territorial, se reflejan los resultados emitidos en el informe de rendición de cuentas por la Superintendencia de Ordenamiento Territorial, Uso y Gestión del Suelo (2019), responsables de vigilar y controlar los procesos de ordenamiento territorial de todos los niveles de gobierno, y del uso y gestión del suelo, hábitat, asentamientos humanos y desarrollo urbano, los Gobiernos Autónomos Descentralizados municipales y metropolitanos dentro del marco de sus competencias, el 35\% ejecuta evaluaciones, actualiza y alinea su instrumento de planificación, un 17\% toma otras acciones como justificación en documentación, mientras que el $48 \%$ no tiene evaluación y no reportan, accionar que resulta de los gobiernos municipales que no toman el interés necesario que tan importante es aplicar y tener actualizado un modelo de gestión que permite obtener una administración territorial eficiente, y por ende, permite alcanzar porcentajes de calificación óptimos.

Realizamos un análisis de los resultados obtenidos de las encuestas aplicadas a los funcionarios del área responsable de planificación en la cual se obtuvieron los siguientes resultados esenciales:

Los funcionarios responsables del área/unidad de planificación encuestados demuestran que se debe mejorar la eficiencia de los modelos de gestión institucionales aplicados en un $74 \%$, se debe trabajar en la definición de funciones, responsabilidades y compromisos, mientras que el $26 \%$ manifiesta que en la actualidad los resultados arrojados son eficientes y suficientes; estos resultados una vez más reflejan que a pesar de la parte documental que mantienen un modelo de gestión por procesos, 2 de 7 municipios atraviesan un cambio de lo tradicional a un modelo actualizado que implica la consideración de herramientas de evaluación como un GPR, pero que ningún gobierno seccional aun no lo dispone. A pesar que los Gad Municipales aplican un modelo de gestión al sector público por procesos enlazado con resultados, es evidente que no existe la eficiencia y satisfacción que haya llegado a su punto de resultados de coadyuvar el desarrollo por parte los usuarios internos (funcionarios) y externos (ciudadanía) de los servicios prestados, de la cultura organizacional y el alcance de los objetivos institucionales.

Por otro lado, los responsables del área de planificación mencionan que un 69\% de los Gobiernos Municipales no priorizan su accionar ante la ejecución eficiente de los modelos de gestión por temas de planificación, definición y responsabilidades asignadas en sus instrumentos de trabajo 
descartando el impacto que genera una articulación con los diferentes niveles de gobierno en beneficio territorial.

En este sentido, a más de la normativa el impacto de la eficiencia de los modelos de gestión en términos de financiamiento a nivel general el Banco Central de Ecuador, citado por Fárez et al. (2014) "los Gobiernos Municipales ecuatorianos recibieron ingresos próximos a los USD 2.000 millones por concepto de asignaciones desde el Gobierno Central, en comparación con los USD 1.156 millones que recibieron en el 2008, lo que implica un incremento anual del 10\% durante los últimos años" Ministerio de Finanzas (2014). En cuanto a gastos, los gobiernos seccionales municipales "destinan cerca del $80 \%$ a inversión; en los últimos años se han registrado altos niveles en los gastos de inversión, pasando de USD 1.244 millones en el 2008 a USD 2.009 millones en el 2013", acotando que a partir de la implementación de un sistema de información del 2009 se ha presentado cambios significativos en la administración y ejecución de los gobiernos seccionales.

Otro factor a considerar que influye en la eficiencia como mejora continua en los modelos de gestión está estrechamente relacionado con el porcentaje de las NBI (Tabla 2), según los datos tomados del 2010 de las encuestas del Instituto Nacional de Estadísticas y Censos, la pobreza por necesidades básicas insatisfechas, la provincia de Cotopaxi presenta una tasa promedio del $81,73 \%$ de los siete cantones, mientras que el $17.93 \%$ de las personas no son pobres e decir cuentan con todos los servicios básicos, sociales y equipamiento correspondientes.

Tabla 2.

Estadísticas de las Necesidades Básicas Insatisfechas

\begin{tabular}{|l|l|l|l|l|l|l|}
\hline Nombre de cantón & NO POBRES & POBRES & Total & NO POBRES & POBRES & Total \\
\hline LATACUNGA & 59.313 & 108493,00 & 167806,00 & $35,3 \%$ & $64,7 \%$ & $100,0 \%$ \\
\hline LA MANA & 11.590 & 30361,00 & 41951,00 & $27,6 \%$ & $72,4 \%$ & $100,0 \%$ \\
\hline PANGUA & 2.177 & 19688,00 & 21865,00 & $10,0 \%$ & $90,0 \%$ & $100,0 \%$ \\
\hline PUJILI & 8.455 & 60432,00 & 68887,00 & $12,3 \%$ & $87,7 \%$ & $100,0 \%$ \\
\hline SALCEDO & 14.284 & 43664,00 & 57948,00 & $24,6 \%$ & $75,4 \%$ & $100,0 \%$ \\
\hline SAQUISILI & 3.962 & 21321,00 & 25283,00 & $15,7 \%$ & $84,3 \%$ & $100,0 \%$ \\
\hline SIGCHOS & 1.371 & 20515,00 & 21886,00 & $6,3 \%$ & $93,7 \%$ & $100,0 \%$ \\
\hline
\end{tabular}

Fuente: Instituto Nacional de Estadísticas y Censos

En la siguiente Figura 3 se evidencia la tendencia de las necesidades insatisfechas con los resultados de los modelos de gestión. 


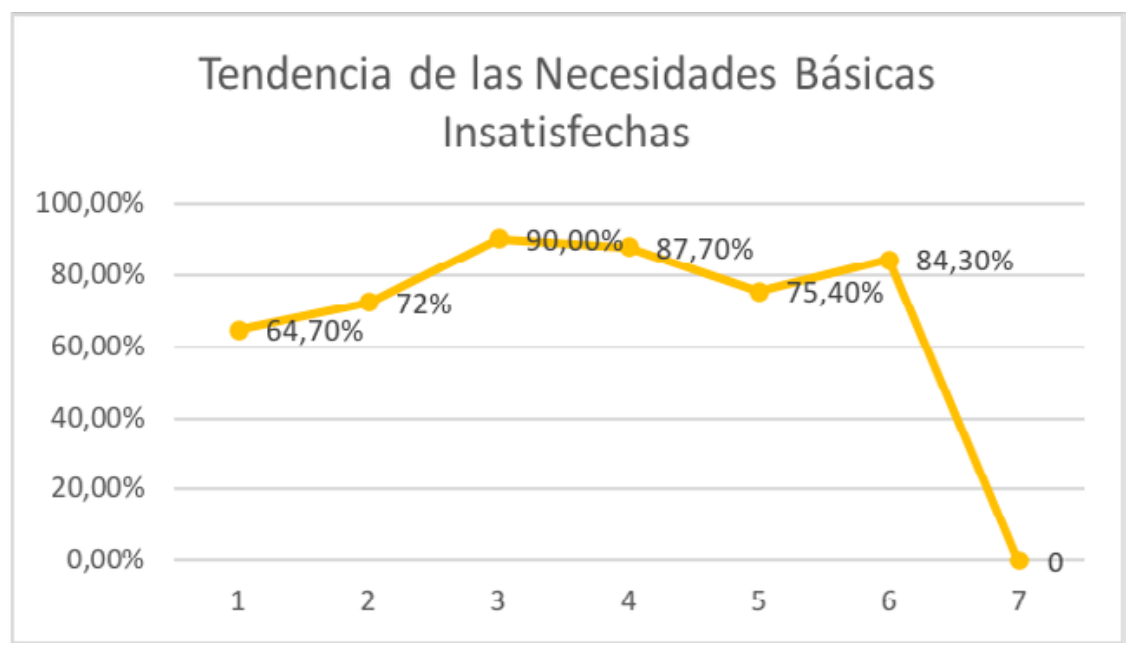

Figura 3. Importancia de un modelo de gestión

Fuente: elaboración propia

La realidad de las Necesidades Básicas Insatisfechas radica en las acciones que debe considerar y priorizar cada Gobierno Municipal en territorio, en donde un modelo de gestión correctamente aplicado es la herramienta estratégica que reduce el porcentaje de las NBI y aumenta la satisfacción de los usuarios por los servicios prestados. En la actualidad, el Gad Municipal de Sigchos, a pesar que en el 2010 presentó un índice del 93.7\% más representativo en necesidades, se entiende que las herramientas de planificación, ejecución y seguimiento no fueron los adecuados; sin embargo, al presente, Sigchos y Saquisili atraviesan un proceso de cambio de modelos tradicionales a modelos de gestión por procesos con enfoques de mejora continua.

De tal manera que algunas instituciones, incluyendo las entidades públicas, han agregado nuevas herramientas de administración pública que buscan optimicen recursos las empresas. Los municipios, al ejecutar el modelo administrativos por procesos, podrán reflejar estrategias de mejora continua para fortalecer la administración pública acorde a sus competencias, a la vez permitirá mejorar los servicios públicos logrando eficiencia y eficacia en el marco institucional.

No obstante, si los Gobiernos Municipales se resisten a los cambios de los modelos de gestión tradicionales, su accionar permanecerá conexa a las funciones, la importancia de un modelo de gestión por procesos si converge a generar servicios y/o productos enfocados a cubrir las necesidades de los ciudadanos de manera productiva.

Con el objetivo de promover acciones de mejoramiento continuo de servicios públicos en concordancia con la constitución en el art. 227, se expresa la nueva administración pública, su importancia y la relación con la Carta Iberoamericana de Calidad en la Gestión Pública que detalla "la administración pública constituye un servicio a la colectividad que se rige por los principios de eficiencia, eficacia, calidad, jerarquía, desconcentración, descentralización, coordinación, participación, planificación, transparencia y evaluación"; centrada en el prestación de servicios al ciudadano y una gestión de resultados. 
Maldonado (2013) focaliza la importancia del fortalecimiento de los Gobiernos Municipales para el desarrollo equitativo, solidario y equilibrado en todo el territorio del acercamiento de la administración a la ciudadanía y el ejercicio de los derechos de participación con todos los actores de la población.

A la vez, se convierten en el eje del progreso respondiendo el desarrollo cantonal, la igualdad de oportunidades y la satisfacción de las necesidades básicas de forma eficiente y eficaz que requieren también un dinamismo con un enfoque basado en procesos para la efectividad de su gestión (Maldonado, 2013).

De acuerdo a esto tienen una importante función los Gobiernos Municipales en el ámbito político y administrativo encaminados a responder desafíos de la dinámica social que requieren ajustes estructurales y a la vez tienen como misión reducir el impacto de as necesidades insatisfechas de la población de un marco territorial bajo los principios de la productividad.

Las estrategias (Figura 4) son modelos de excelencia que permiten obtener ventajas y cumplir los objetivos institucionales, a la vez son metodologías y acciones de aplicación que aportan a la toma acertada de decisiones. La consecución de un modelo por resultados se base en la mejora continua mediante la incorporación de acciones por lo que se plantea un mapa estratégico con cuatro procesos o punto de perspectiva en procesos, talento humano, financiero y ciudadano, y tres ejes enfocados en la parte social, económico, control y seguimiento.

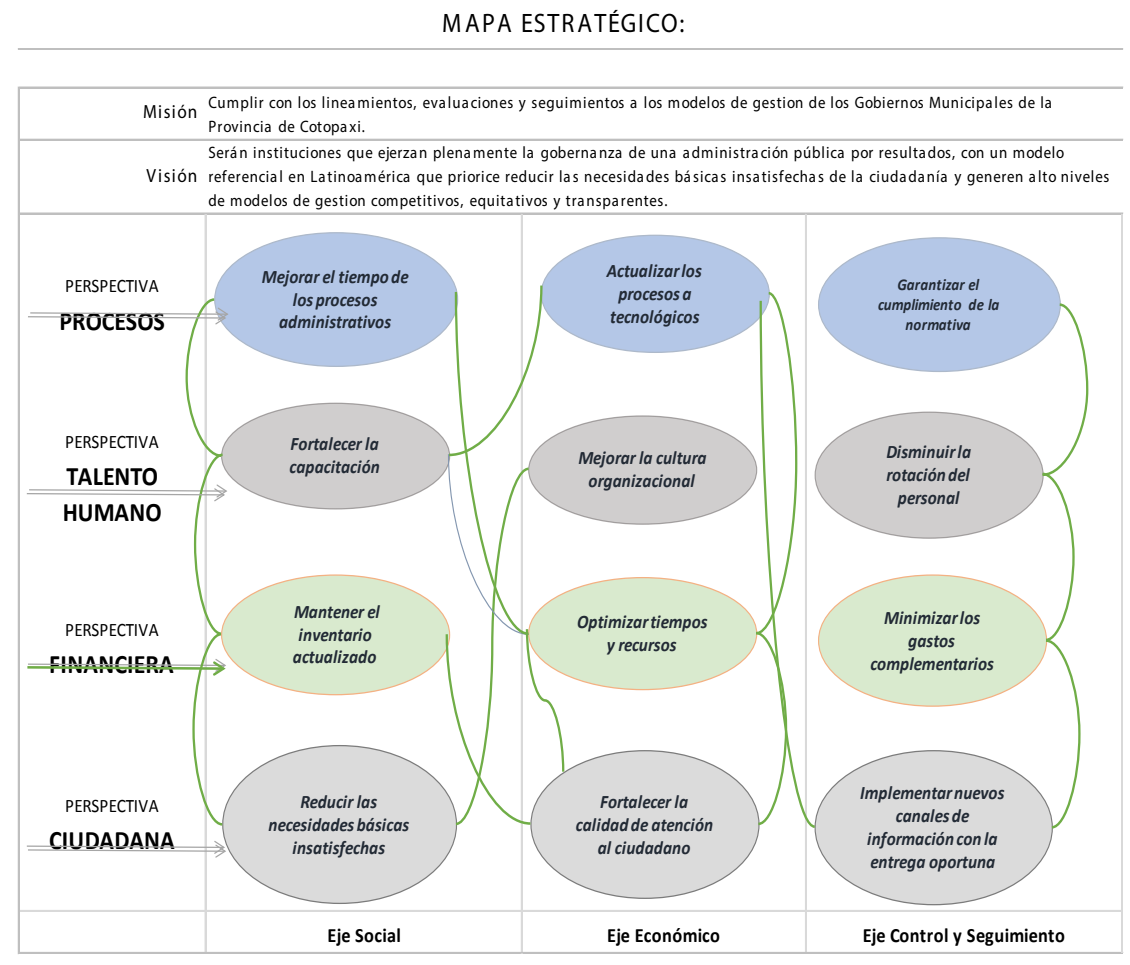

Figura 4. Propuestas de acciones estrategias

Fuente: elaboración propia 
A través del presente mapa estratégico, que es una presentación visual de las estrategias trasversales enlazadas a los Gobiernos Municipales, se reflejan los vínculos estrechos con cada uno de las perspectivas y ejes que permiten articular esfuerzos e iniciativas institucionales para mejorar la prestación de los servicios públicos territoriales. Además, el control y seguimiento ayudará a implementar canales y entrega oportuna de la información con proceso de descentralización.

\section{Discusión}

Al analizar la forma de ejecución de los Gobiernos Municipales, el cumplimiento de cada uno de sus objetivos institucionales es mejor exigir se impulsen en generar recursos y servicios públicos que solventen las demandas de los ciudadanos de manera oportuna, mejorando la administración pública con el impacto de la reducción de los índices de la necesidades básicas insatisfechas, colaboración de los funcionarios de aplicar el modelo de gestión en procesos que generen resultados trascendentales que permiten una mejor calidad de vida y el bienestar social.

A pesar que la asignación presupuestaria es otro rol que influyen en los modelos de gestión para su cumplimiento, debe unir esfuerzo para articular con los diferentes niveles de gobierno y buscar las estrategias necesarias o convenios interinstitucionales en beneficio de la ciudadanía territorial.

\section{Conclusiones}

La presente investigación permitió analizar la importancia de los modelos de gestión como estrategia de mejora continua. A través de los resultados obtenido se ha logró identificar que en los modelos de los Gobiernos Municipales de la provincia de Cotopaxi el $74 \%$ expresa que se debe tomar acciones para mejorar los modelos tradicionales a modelos restructurados que propongan estrategias vinculadas a la articulación y progreso a fin de reducir las debilidades diagnosticadas en el desconocimiento de los lineamiento de la ejecución y desactualización de los modelos que no permiten el incremento de personas que accedan al buen vivir con todo los servicios y equipamientos necesarios.

En la administración de los Gobierno Municipales se debe actuar en virtud que el $48 \%$ no tiene evaluación y no reportan acciones que impiden que tomen el interés necesario de aplicar y tener actualizado un modelo de gestión focalizado a obtener una administración territorial eficiente, y por ende, no permite alcanzar porcentajes de calificación óptimos; un modelo de gestión correctamente aplicado es la herramienta estratégica que reduce el porcentaje de las NBI y aumenta la satisfacción de los usuarios por los servicios prestados.

Finalmente, se construyó un mapa estratégico que constituye una herramienta especialmente necesaria que prioriza las acciones en base a las perspectivas y los ejes para el desarrollo sostenible y sustentable de cada uno de uno de los territorios cantonales identificados a generar objetivos estratégicos para cada misión de trabajo, resaltando un vez más la importancia de trascender con los modelos de gestión tradicionales a innovadores coadyuvados con la eficiencia 
permite una administración pública por resultados a fortificar la duración del desarrollo integral de un territorio y solventar las necesidades básicas de los diferentes actores territoriales. 


\section{Referencias}

Asamblea Nacional (2015). Código Orgánico Organización Territorial Autonomía Descentralización. https://www. finanzas.gob.ec/wp-content/uploads/downloads/2012/09/CODIGO ORGANIZACION TERRITORIAL.pdf

Bañón i Martínez, R. (1993). La modernización de la Administración Pública española. Balance y perspectivas. Política Y Sociedad, 13, 9-20. Recuperado a partir de https://revistas.ucm.es/index.php/POSO/article/ view/POSO9393220009A

Chávez Camacho, K \& Otavalo Quizhpe, D. (2018). Evaluación al ciclo presupuestario del Gobierno Autónomo Descentralizado Municipal del cantón Cuenca, período 2016.

Chilán Robles, S., Chilán Indacochea, J., Marcillo Indacochea, M., \& Álvarez Indacochea, A. (2016). Modelo de gestión administrativa para optimizar el desempeño de los gobiernos autónomos descentralizados parroquiales. 3C Empresa. Investigación Y Pensamiento Crítico, 5(2), 99 - 113. https://doi.org/10.17993/ 3cemp.2016.050226.99-113

Fárez Plasencia, M. I., \& Quinde Lituma, M. E. (2014). Análisis de la eficiencia del gasto de inversión de los gobiernos autónomos descentralizados municipales del Ecuador para el año 2014: estimación de una frontera estocástica.

Fernández, A. (2001). El Balanced Scorecard. Revista de antiguos alumnos del IESE, 81, 83.

Flores Barcia, L. D. (2017). Diseño y aplicación de un modelo de excelencia y mejora continua en un gobierno autónomo descentralizado (Master's thesis, Espol). http://www.dspace.espol.edu.ec/xmlui/bitstream/ handle/123456789/38586/D-CD102527.pdf?isAllowed=y\&sequence=-1

Hernández Sampieri, R., Fernández Collado, C., \& Baptista Lucio, M. (2014). Metodologia de la Investigación. México: McGRAW-HILL / INTERAMERICANA EDITORES, S.A. DE C.V.

Hervas, J. A. V. (1977). Guía para la ejecución de estudios de organización y métodos: la encuesta administrativa. Universidad Central del Ecuador, Facultad de Ciencias Administrativas.

Instituto Nacional de Estadísticas y Censos. (s.f). Pobreza por Necesidades Básicas Insatisfechas. https://www. ecuadorencifras.gob.ec/pobreza-por-necesidades-basicas-insatisfechas/

Izquierdo Acosta, R. D. P. (2015). Modelo de gestión administrativa para el gobierno autónomo descentralizado parroquial rural de Guayllabamba, distrito metropolitano de Quito, provincia de Pichincha (Bachelor's thesis, Quito: UCE.). 
López Cubino, R. (2001). Modelos de Gestión de Calidad. Ministerio de Educación, Cultura y Deporte del año 2001, "Modelo Europeo de Excelencia”. https://www.jesuitasleon.es/calidad/Modelos\%20de\%20gestion\%20de\%20calidad.pdf

Maldonado Vizcaíno, F. V. (2013). Propuesta de implementación del modelo de gestión por procesos para mejorar la eficiencia y eficacia organizacional del Gobierno Autónomo Descentralizado de Sigchos.

Peñeherrera Cabezas, J. (2016). La planificación nacional en Ecuador: planes de desarrollo y ordenamiento territorial y el sistema de seguimiento y evaluación SIGAD. Ciencia Unemi, 12, 3-13. Revista Ciencia UNEMI, 9(21), 168-179. https://dialnet.unirioja.es/servlet/articulo?codigo=6018808

Pepper Bergholz, S. (2011). Definición de gestión por procesos. Medwave 11(05). https://doi.org/10.5867/medwave.2011.05.5032

Peteiro, D. R. (s.f). Calidad y Excelencia Empresarial. Sinapsys Business Solutions. https://www.sinap-sys.com/ es/content/calidad-y-excelencia-empresarial

PRESIDENCIA DE LA REPUBLICA DEL ECUADOR. (2010). CODIGO ORGANICO DE ORGANIZACION TERRITORIAL, AUTONOMIA Y DESCENTRALIZACION. (última modificación: 16-ene.-2015). https://www. defensa.gob.ec/wp-content/uploads/downloads/2016/01/dic15 CODIGO-ORGANICO-DE-ORGANIZACION-TERRITORIAL-COOTAD.pdf

Programa de las Naciones Unidas para el Desarrollo - PNUD (s.f). OBJETIVOS DE DESARROLLO SOSTENIBLE.

Sistema Nacional de Información. (2009). Un nuevo modelo de gestión de la información Nacional.

Secretaría Nacional de Planificación y Desarrollo - SENPLADES. (2016). Sistematización de Buenas Prácticas Institucionales en el marco del Modelo de Gestión del Conocimiento. https://www.planificacion.gob.ec/ wp-content/uploads/downloads/2016/07/Sistematizacion-de-Buenas-Practicas-Institucionales-en-el-marco-del-Modelo-de-Gestion-del-Conocimiento.pdf

Scharager, J \& P. Reyes. (2001). Muestreo no probabilístico. Pontificia Universidad Católica de Chile, Escuela de Psicología (2001): 1-3.

Superintendencia de Ordenamiento Territorial, Uso y Gestión de Suelo - SOT. (2015). Actualización del Plan de Desarrollo y Ordenamiento Territorial Cotopaxi 2025.

Vidal Arizabaleta, E. (2004). Diagnostico organizacional: evaluación sistemática del desempeño empresarial. Colección Textos Universitarios. 
Waissbluth, M., \& Larrain, F. (2020). Modelos de gestión pública: implicancias para la planificación, evaluación y control de gestión del Estado. Un mejor Estado para Chile. Propuestas de Modernización y Reforma, 541-559. http://www.sistemaspublicos.cl/wp-content/uploads/2018/02/Modelos-de-gesti\%C3\%B3n-p\%C3\%BAblica-implicancias-para-la-planificaci\%C3\%B3n-evaluaci\%C3\%B3n-y-control-de-gesti\%C3\%B3n-del-Estado.pdf 


\section{Copyright (c) 2021 Carmen Marlene Choloquinga Escobar}

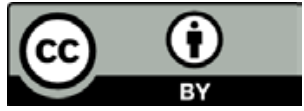

Este texto está protegido bajo una licencia internacional Creative Commons 4.0.

Usted es libre para Compartir - copiar y redistribuir el material en cualquier medio o formato - y Adaptar

el documento - remezclar, transformar y crear a partir del material-para cualquier propósito, incluso para fines comerciales, siempre que cumpla las condiciones de Atribución. Usted debe dar crédito a la obra original de manera adecuada, proporcionar un enlace a la licencia, e indicar si se han realizado cambios. Puede hacerlo en cualquier forma razonable, pero no de forma tal que sugiera que tiene el apoyo del licenciante o lo recibe por el uso que hace de la obra.

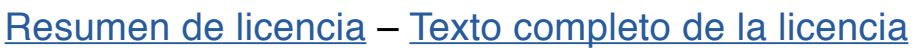

